\title{
VIBRATION-POWERED SENSING SYSTEM FOR ENGINE CONDITION MONITORING
}

\author{
A.S. Weddell, G.V. Merrett, S. Barrow, B.M. Al-Hashimi \\ Electronics and Computer Science, University of Southampton, Southampton, SO17 1BJ, UK \\ \{asw,gvm,sb35g08,bmah\}@ecs.soton.ac.uk Tel. +44(0)238059 4996 Fax. +44(0)23 80592901
}

Keywords: energy harvesting, wireless sensing, system design, condition monitoring

\begin{abstract}
Condition monitoring is becoming an established technique for managing the maintenance of machinery in transport applications. Vibration energy harvesting allows wireless systems to be powered without batteries, but most traditional generators have been designed to operate at fixed frequencies. The variety of engine speeds (and hence vibration frequencies) in transport applications therefore means that these are not usable. This paper describes the applicationdriven specification, design and implementation of a novel vibration-powered sensing system for condition monitoring of engines. This demonstrates that, through careful holistic design of the entire system, condition monitoring systems can be powered solely from machine vibration, managing their energy resources and transmitting sensed data wirelessly.
\end{abstract}

\section{Introduction}

Condition-based maintenance programmes are used to monitor the health of engines and mechanical parts in transport applications [4], and are becoming increasingly common. Condition monitoring facilitates the early detection of faults and enables preventative action to be taken, thereby avoiding unplanned machine failure putting craft or vehicles out of action [5]. Online condition monitoring systems are permanently installed and operate continuously, and are usually hard-wired (meaning that cabling must be installed). Although the initial installation costs of a wireless sensor system are likely to be lower, they are typically unattractive due to a need for battery replacement when depleted, imposing a further maintenance cost. This problem has been partially addressed by energy harvesting sensor nodes (e.g. for use on a helicopter [6]), but have so far been designed to harvest energy from fixed-frequency vibrations. Recent research has proposed vibration harvesters that are able to adjust their resonant frequency [7], but there is a need to consider the impact of this at the system level (rather than simply adding an adaptive generator to an existing system).

This paper describes a new wireless online condition monitor which is powered solely from engine vibrations (hence not requiring either batteries or cabling). The system was developed by 1) considering the dynamics of the vibration of a real engine, 2) devising a system architecture which delivers high energy efficiency and effective cold-starting of a sensor node, and 3) designing a method for adjusting to changes in vibration frequency in a real-world environment.

The application case study used in this paper is one of condition monitoring on the engine of a "ro-ro" vehicle ferry. The system is intended to monitor the vibration of the engine by periodically taking a snapshot of the acceleration levels and performing an FFT to compute vibration levels. The temperature of the test location is also monitored. By analysing long-term trends or detecting unusual values, early indication of machinery problems (such as bearing failure) can be given, allowing maintenance to be carried out before the machinery actually fails. The system is entirely powered by the vibration of the engine and is able to cold-start.

This paper reports the first vibration-powered condition monitoring system able to track varying frequency vibrations, and adapt to changing energy resources. This makes it applicable to applications where engine speeds and loadings (hence vibration frequencies and amplitudes) are changeable. This allows it to intelligently manage sampling, computation and communication activity to control energy consumption.

\section{Vibration Characteristics of a Ferry Engine}

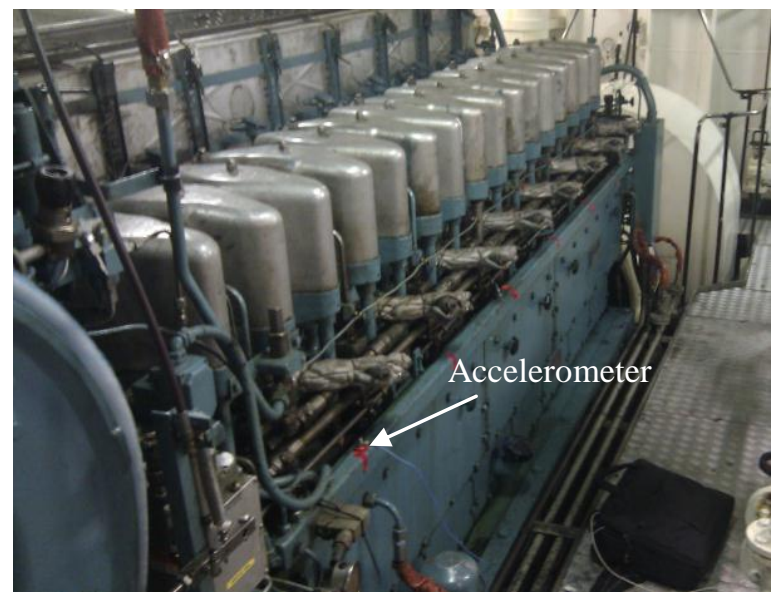

Figure 1: Accelerometer mounted on ferry engine (8-cylinder marine diesel, driving a Voith-Schneider propulsion unit)

In transport applications, the frequency and amplitude of engine vibrations are dependent on their activity. This differs 
from established applications for vibration energy harvesting, where frequencies are generally fixed (such as machinery driven by mains-powered motors). To sample the vibration characteristics of ship engines, a PCB Piezotronics 354C03 tri-axial accelerometer was attached to the main engine of a ferry (Fig. 1) on two occasions (different ferries operating on the same route) using a magnetic mount. It was connected through a PCB Piezotronics 480B21 signal conditioner and Keithley KUSB-3102 USB data logger to a laptop computer and sampled at $1024 \mathrm{~Hz}$ to monitor its characteristics during return crossings. The engine is an 8-cylinder FHD240 Wartsila diesel, connected to a Voith-Schneider propulsion arrangement. The engine is typically operated at speeds between $350-750$ RPM ( 715 RPM at sea to $\sim 350$ RPM when idling in port).

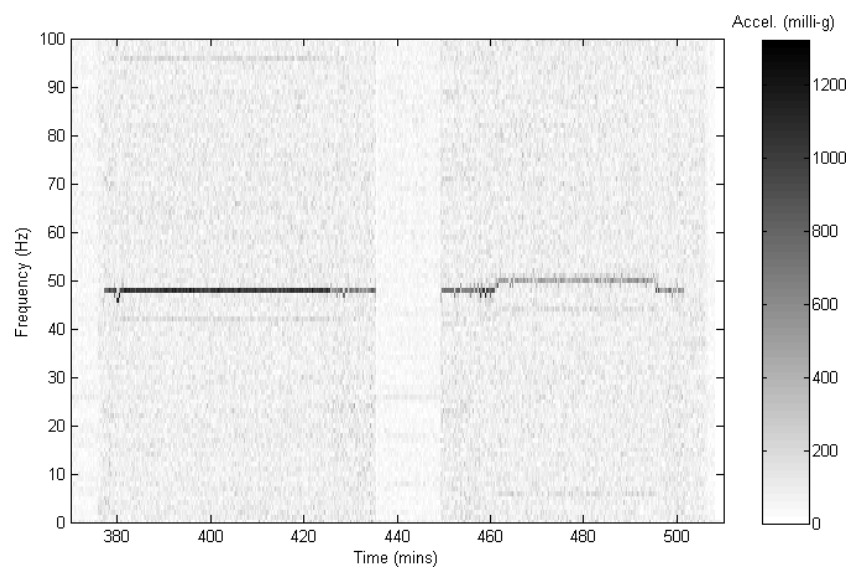

Figure 2: FFT of transverse-axis data from ship engine (2 crossings). Dominant frequency corresponds to $4 \mathrm{x}$ engine rev. speed (8-cylinder engine). 1 ' $\mathrm{g}$ ' $=9.81 \mathrm{~ms}^{-2}$.

The data was processed in MATLAB using a 1024-point $1 \mathrm{~s}$ FFT, providing a $1 \mathrm{~Hz}$ frequency resolution. Analysis of the data revealed that the transverse direction (Fig. 2) had highest vibration levels, and the dominant frequencies (shown in Table 1) corresponded to the engine's firing frequency (i.e. four times the engine revolution speed), except in the case of ferry 1 while idling where the significant component was double this frequency. The raw data for these tests can be downloaded from the Energy Harvesting Network's Data Repository [1].

From these results, outline specifications for the vibration energy harvester were generated (Table 2), which allow a $\pm 10 \%$ margin on the upper and lower operating frequencies. The generator was manufactured, and was used to harvest vibrations at these frequencies and amplitudes. It is of a similar construction and incorporates a mechanical tuning strategy described in [3]. The maximum power output (through an optimal resistive load) from the energy harvester is up to $10 \mathrm{~mW}$, which imposes challenges on the design of the rest of the system.

\begin{tabular}{|l|l|l|l|l|l|l|}
\hline \multirow{2}{*}{ Ferry } & \multicolumn{2}{|c|}{$\begin{array}{c}\text { Speed 1 } \\
(\sim 350 \text { RPM })\end{array}$} & \multicolumn{2}{c|}{$\begin{array}{c}\text { Speed 2 } \\
(\sim 715 \text { RPM })\end{array}$} & \multicolumn{2}{c|}{$\begin{array}{c}\text { Speed 3 } \\
(\sim 750 \text { RPM })\end{array}$} \\
\cline { 2 - 7 } & $\begin{array}{l}\text { Freq. } \\
(\mathrm{Hz})\end{array}$ & $\begin{array}{l}\text { Ampl. } \\
\left(\mathrm{mg}_{\mathrm{pk}}\right)\end{array}$ & $\begin{array}{l}\text { Freq. } \\
(\mathrm{Hz})\end{array}$ & $\begin{array}{l}\text { Ampl. } \\
\left(\mathrm{mg}_{\mathrm{pk}}\right)\end{array}$ & $\begin{array}{l}\text { Freq. } \\
(\mathrm{Hz})\end{array}$ & $\begin{array}{l}\text { Ampl. } \\
\left(\mathrm{mg}_{\mathrm{pk}}\right)\end{array}$ \\
\hline 1 & 47 & 100 & 47 & 700 & Not tested \\
\hline 2 & \multicolumn{2}{|c|}{ V. low ampl. } & 48 & 950 & 50 & 450 \\
\hline
\end{tabular}

Table 1: Typical vibration levels on engines of two ferries.

\begin{tabular}{|l|l|}
\hline Min. frequency & $42 \mathrm{~Hz}$ \\
\hline Max. frequency & $55 \mathrm{~Hz}$ \\
\hline Max. amplitude & $1000 \mathrm{mg}_{\mathrm{pk}}$ \\
\hline Max. power output (loaded) & $10 \mathrm{~mW}$ \\
\hline Min. voltage output (loaded, speed 2) & $5 \mathrm{Vp}-\mathrm{p}$ \\
\hline
\end{tabular}

Table 2: Specification for tuneable vibration harvester.

\section{System Architecture}

\subsection{Overview}

Due to the variable nature of machinery vibration and the associated power output of energy harvesters, the system architecture (Fig. 3) was designed to accommodate variability in its power supply. It provides a stable DC supply from the unrectified output from the harvester, and supports a) coldstarting, b) overvoltage protection, c) adaptive operation, and d) tracking of frequency changes of the vibration source. By permitting the microcontroller to monitor the store voltage, it allows it to adapt its behaviour to its energy status. The

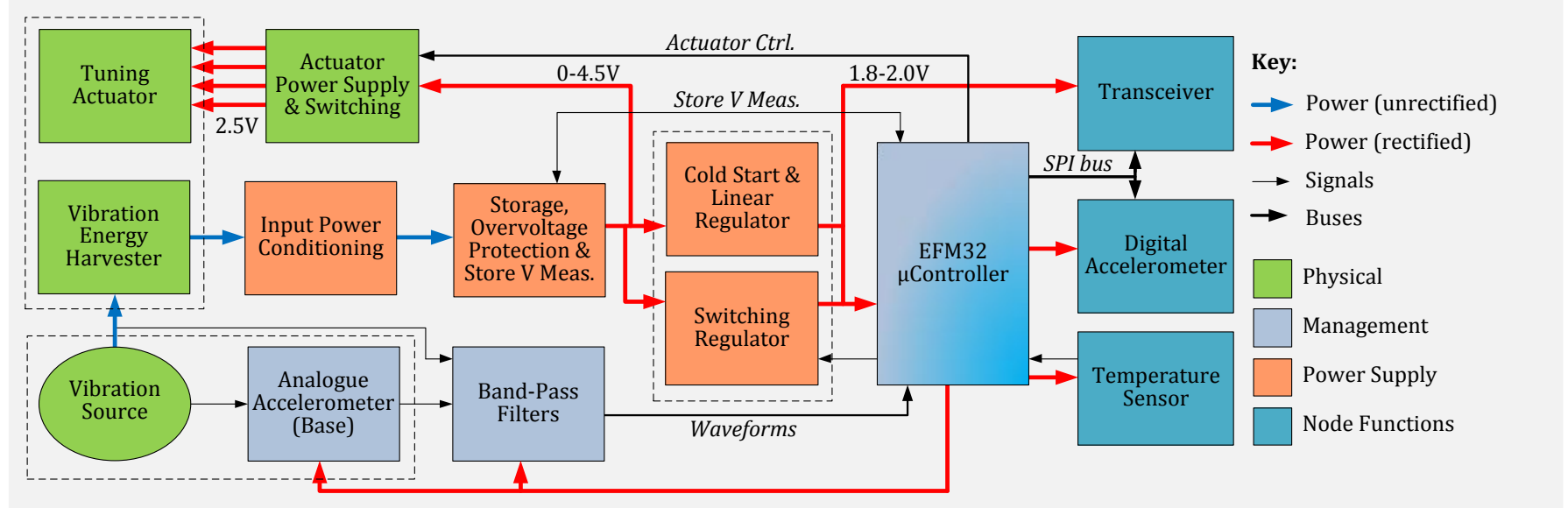

Figure 3: Detailed block diagram of system. Adapted from Holistic Energy Harvesting project [2] 
operation of the sensing system is managed by an EFM32 microcontroller, which interfaces with condition sensors, transmitting data with a sub-GHz radio transceiver. The system is designed to monitor the frequency of the source vibration and react to changes by adjusting the actuator; this is itself an energy-intensive activity that must be done carefully to avoid wasting energy through unnecessary actuation or sending the harvester off-resonance. The functionality of each part of the system is described in turn.

\subsection{Vibration Source, Harvester and Input Conditioning}

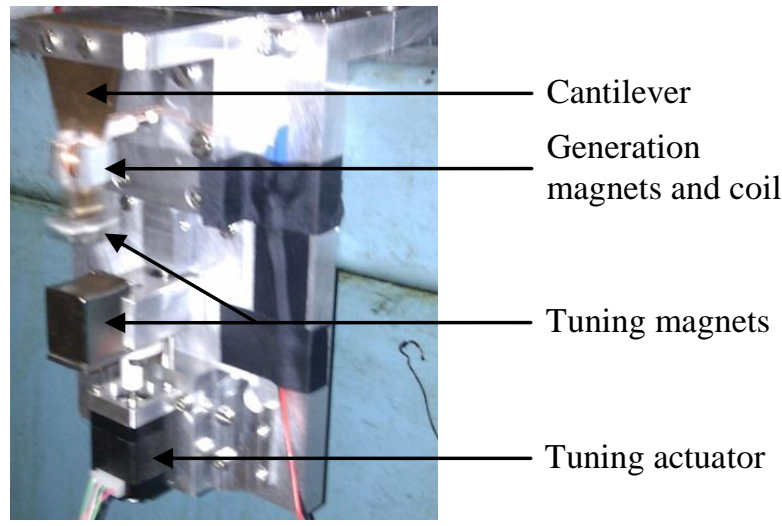

Figure 4: Energy harvester mounted to side of ferry engine.

The harvester is clamped to the side of the engine (Fig. 4), with it being designed to exploit oscillations in the transverse direction. The construction of the harvester includes a $\mathrm{BeCu}$ cantilever, and two sets of $\mathrm{NdFeB}$ magnets, which are used for tuning and for power generation. The harvester is tuned by adjusting the distance between the tuning magnets (and hence the force acting on the cantilever); this is carried out by the tuning actuator. A coil, constructed from $60 \mu \mathrm{m}$ diameter copper, with outer diameter $10 \mathrm{~mm}$, inner diameter $1 \mathrm{~mm}$, and thickness $4 \mathrm{~mm}$ was used for energy generation. The input power conditioning arrangement is a bridge rectifier constructed from low $\mathrm{V}_{\mathrm{f}}$ Schottky barrier diodes.

\subsection{Storage, Overvoltage, and Voltage Measurement}

The primary considerations for selecting the energy storage module is that it should be able to 1) accept the bursty input of harvested energy from the energy harvester and 2) supply the bursty power consumption of the node and, particularly, the tuning actuator, without excessive voltage depression, and 3) store sufficient energy for the tuning actuator to move across its full range. Given the maximum drive speed of 3.4 $\mathrm{mm} / \mathrm{s}$ (340 steps/s), this takes approximately $1.8 \mathrm{~s}$ to move across the full $6 \mathrm{~mm}$ actuation range. With an average power consumption of $625 \mathrm{~mW}$, this means that actuation across the full range consumes approx. $1.1 \mathrm{~J}$.

In view of the intermittent and bursty nature of energy generation and usage, supercapacitors were identified as a suitable option for energy storage. Given that they are commonly available rated at up to $2.3 \mathrm{~V}$, and most low-power devices operate down to 2.0 or $1.8 \mathrm{~V}$, a series combination of two supercapacitors offers a maximum 4.6 V. Sizing of the supercapacitor combination is dominated by the energy consumed by tuning. Assuming that the supercapacitor combination could discharge from $4.5 \mathrm{~V}$ to $2.5 \mathrm{~V}$ to provide the full range of actuation, it may be calculated that a minimum $157 \mathrm{mF}$ is required. To give flexibility and allow for other system operations, a $500 \mathrm{mF} 4.6 \mathrm{~V}$ supercapacitor combination has been used, formed of 2 x 1 F 2.3 V Panasonic Gold supercapacitors in series.

To limit the supercapacitors voltage to $4.5 \mathrm{~V}$ max., a voltage detector turns on an LED when the voltage exceeds this threshold. This 'overvoltage spillover' arrangement effectively acts as a visual indicator of when the energy store has become full, while limiting the voltage to $4.5 \mathrm{~V}$. Separately, a voltage measurement circuit uses a voltage divider and unity gain buffer, allowing the microcontroller to observe the voltage of the store.

\subsection{Cold Start and Output Power Conditioning}

Without a cold-start arrangement, the microcontroller will attempt to start up when the store voltage is too low, entering a state where it is unable to start up but drawing a substantial amount of current. Given the limited power that can be harvested, this means that the system would be locked into this state and never start up. For this reason, a cold-start arrangement incorporating two CMOS voltage detector ICs has been designed. It allows the system to start at $2.0 \mathrm{~V}$ and cuts the supply when the store voltage drops below $1.8 \mathrm{~V}$.

In order to conserve energy, and reduce the power demand of the system, wireless sensing systems typically spend most of their time (often more than 99\%) in a 'sleep' mode. For this reason, the quiescent power consumption of the system when sleeping is very important. Motivated by this, a two-stage output power conditioning arrangement was devised. This uses a $1.8 \mathrm{~V}$ linear regulator, and a separate $2.0 \mathrm{~V}$ switching step-down regulator which is enabled by an output from the microcontroller. By using this arrangement, the naturally lower quiescent current draw of the linear regulator is exploited when the device is sleeping, and the higher efficiency of the switching regulator can be used when the system is active and the store voltage is sufficient to permit operation of the switching converter. In this way, the quiescent power consumption of the system is kept to a minimum, and stable start-up is assured. Alternative topologies incorporating buck-boost converters were considered but ruled out due to their lower efficiency levels and higher quiescent current draw.

The effect of these design decisions is to deliver a power supply to the microcontroller and its peripherals that steps the supercapacitor voltage down to either $1.8 \mathrm{~V}$ or $2.0 \mathrm{~V}$ (dependent on whether the switching regulator is active). Including the cold start arrangement, this part of the system has a designed minimum quiescent current of approx. $6 \mu \mathrm{A}$ (when operating using only the linear regulator) and a 
designed efficiency of approx. 90\% (when the switching regulator is active and supplying $>10 \mathrm{~mA}$ ).

\subsection{Actuator, Actuator Power Supply and Control}

A low-voltage actuator (Haydon E21H4U-2.5-900) is used to perform tuning actuation. This device draws $490 \mathrm{~mA}$ at $2.5 \mathrm{~V}$, and has a linear travel of approx. $0.01 \mathrm{~mm} / \mathrm{step}$; this device was selected due to its availability and its ability to provide the required tuning force. The resonant frequency of the generator varies between $42 \mathrm{~Hz}$ and $55 \mathrm{~Hz}$ with approx. $6 \mathrm{~mm}$ of movement from the actuator.

To provide the substantial currents required with good levels of efficiency, a Texas Instruments TPS 62006 step-down converter was used. The stepper motor was driven full-step with ramp-up and ramp-down by the microcontroller; power switching was carried out using a FET-based arrangement that required four control lines from the microcontroller. The position of the tuning actuator is monitored using a BEI Sensors resistive linear position sensor. This allows the position of the actuator to be measured and is especially important in cases where the microcontroller has powercycled thus losing its record of the actuator's current position.

\subsection{Analogue Accelerometer and Filters}

A major challenge in this system is in devising an efficient and effective way for the tuned frequency of the generator to follow the frequency of vibrations of the source (in this case, the engine). In the existing system [3], this was a two-phase process. Firstly, the frequency of vibration of the source was coarsely estimated by attaching an analogue accelerometer to the base and connecting it to the ADC of a microcontroller, and moving the actuator to the best estimated position to deliver this resonant frequency. Fine-tuning was then achieved by acting to minimise the phase difference between the generator output and the accelerometer output. However, the system was only applicable to pure sinusoidal vibrations.

In real-world systems, as shown in Fig. 2, vibrations are typically noisy and composed of several harmonics. Using the simplistic algorithms described by Garcia et al. [3] alone results in poor performance as algorithms to detect the dominant frequency and phase difference are also sensitive to the harmonics, resulting in unpredictable behaviour and the inability to tune to the appropriate frequency. An obvious strategy to avoid this problem is to pass the accelerometer output through a band-pass filter, thus only looking at the frequency band that the generator is able to tune across. However, by the nature of the band-pass filter, phase distortion is introduced meaning that the fine-tuning algorithm from Garcia et al. [3] would not work.

To overcome the problems of phase distortion between the filtered accelerometer output and the voltage output from the energy harvester, a band-pass filter with similar properties was also added to the line monitoring the voltage output from the harvester, as shown in Fig. 3. By using the same type of filter for both parts (a Butterworth filter) with the same passband but differing gain, a similar level of phase distortion is introduced to both channels thus allowing the phase difference technique to be used in this real-world application.

\subsection{Radio Transceiver and Digital Accelerometer}

For this system to act as a wireless sensor node for condition monitoring, it is designed to monitor the temperature and vibration of the engine. The microcontroller performs an FFT on raw data obtained from an Analog Devices ADXL345 triaxial digital accelerometer. It also has the capability of acting in an 'alarm mode' by sending an entire FFT less frequently, and forcing transmission of a full FFT in the event of a vibration amplitude threshold being crossed., and also queries the temperature measured by an analogue temperature sensor. The system transmits using a Texas Instruments CC1101 radio transceiver which operates in the $433 \mathrm{MHz}$ ISM band. An SPI interface is shared between the accelerometer and transceiver. The power supply for the accelerometer is supplied directly from an output from the microcontroller, while the transceiver shares the same $1.8 / 2.0 \mathrm{~V}$ power supply as the microcontroller but sleeps regularly (only waking to perform transmissions as commanded by the EFM32).

\section{System Operation}

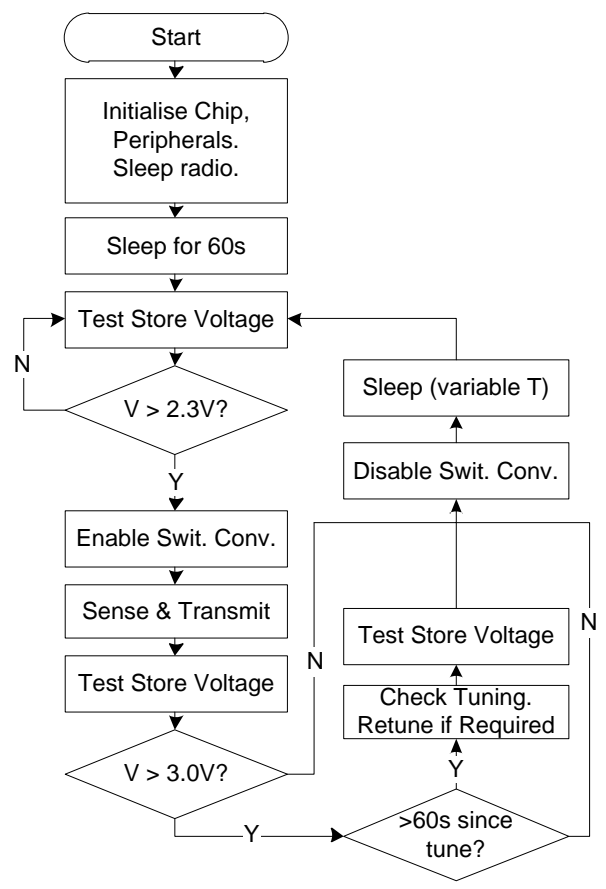

Figure 5: Operating sequence of sensor node.

As described in Section 3, the generator output is conditioned by the bridge rectifier and stored in the supercapacitors. When the supercapacitor voltage exceeds $2.0 \mathrm{~V}$, the cold-start arrangement supplied power to the $1.8 / 2.0 \mathrm{~V}$ line which allows the microcontroller to start up. When the microcontroller starts up, it goes through the steps illustrated in Fig. 5. Namely, on startup it initialises the other devices then immediately returns to sleep. After sleeping for 60 s, it wakes 
up and tests its supply voltage; if this exceeds $2.3 \mathrm{~V}$, the stepdown converter is enabled and the sensors are queried; data is processed and a transmission is performed. Transmissions are received with an evaluation board connected to a PC.

After transmission, if the store voltage is above $3.0 \mathrm{~V}$ the node checks if the tuning of the generator needs to be adjusted, and adjusts the tuning if required. The switching converter is then disabled and the node returns to sleep, for a period dictated by the store voltage. In this way, the node's activity is modulated by the energy stored.

\section{Testing and Results}

\subsection{Modular Testing}

The dual band-pass filters deliver a centre frequency of $48 \mathrm{~Hz}$ and a $3 \mathrm{~dB}$ bandwidth of $15 \mathrm{~Hz}$, and were tested together. For the phase distortion between the two filters, there is a $17^{\circ}$ change across the range $42-54 \mathrm{~Hz}$, and a $3^{\circ}$ change in the $47-$ $50 \mathrm{~Hz}$ central pass band (equating to a $5 \%$ and $0.8 \%$ change in the phase relationship, respectively). The $0.2 x$ filter current draw was $44 \mu \mathrm{A}$ with a $52 \mathrm{~Hz} 5 \mathrm{Vp}$-p input signal.

The bridge-rectifier input power conditioning circuit realised a peak harvester utilisation of $48 \%$ (this is the power stored in the supercapacitor vs. the power through an optimal resistive load). The cold-start arrangement was tested and drew $7.7 \mu \mathrm{A}$ to $9.5 \mu \mathrm{A}$ across the operating voltage range when the switching converter is disabled; the quiescent current is 26 $\mu \mathrm{A}$ when the switching converter is enabled, with an efficiency of approx $86 \%$ when supplying a $10 \mathrm{~mA}$ load. The switching converter for the stepper motor has been tested as being $89 \%$ efficient when supplying a $5 \Omega$ (approx $1.18 \mathrm{~W}$ ).

\subsection{System Testing}

The results of system testing are shown in Fig. 6, where the sensor system was attached to an engine and transmitted an FFT of the measured vibration. The system is able to coldstart and operates autonomously, tracking changes in the frequency of the source vibration. A stable supply is provided to the microcontroller, transceiver, and temperature and vibration sensors. The device performs an FFT on raw data obtained from the 3-axis MEMS accelerometer, performs analysis, and transmits the results wirelessly. The energy cost of a sense-and-transmit cycle is shown in Table 3. The node dynamically manages its energy consumption by adapting its duty cycle; this table shows the energy use for a 10 s senseand-transmit cycle.

\begin{tabular}{|l|ll|}
\hline Phase of Operation & Time & Energy \\
\hline Read accelerometer & $174 \mathrm{~ms}$ & $657 \mu \mathrm{J}$ \\
FFT & $538 \mathrm{~ms}$ & $4870 \mu \mathrm{J}$ \\
Transmission & $778 \mathrm{~ms}$ & $4360 \mu \mathrm{J}$ \\
Sleep & $10 \mathrm{~s}$ & $37 \mu \mathrm{J}$ \\
Total & $11.49 \mathrm{~s}$ & $9.92 \mathrm{~mJ}$ \\
\hline
\end{tabular}

Table 3: Energy use for a $10 \mathrm{~s}$ sense-and-transmit cycle (this is dynamically adjusted by the sensor node).
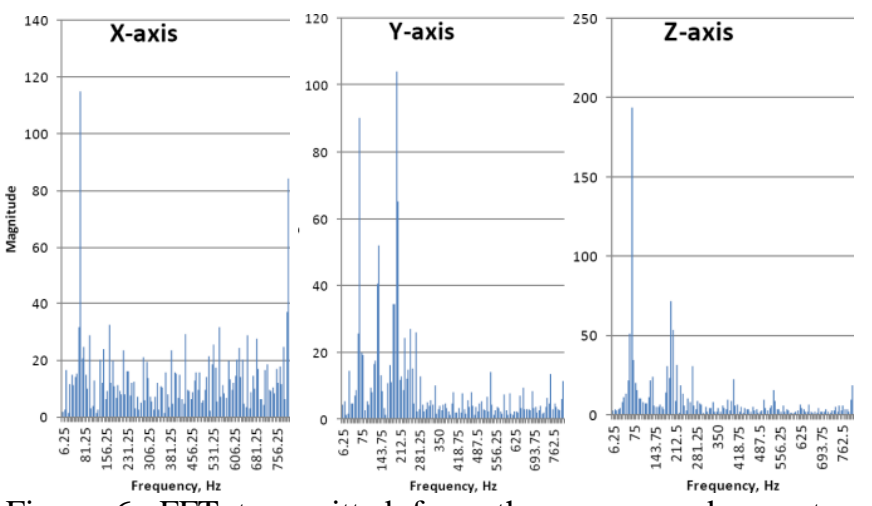

Figure 6: FFT transmitted from the sensor node system, demonstrating system operation on an engine.

\section{Conclusion}

In this paper, we have reported on a novel self-powered condition monitor that is powered by harvesting energy from vibrations in a real environment. The system dynamically tracks the changing vibration frequencies to maximise harvested energy. By first considering the dynamics of the application and holistically designing the rest of the system, an efficient prototype has been produced. Next, the complete system will be tested on a ferry for an extended period to verify its reliability in an operational setting.

\section{Acknowledgements}

This work was supported by the Engineering and Physical Sciences Research Council (EPSRC) under grant number EP/G067740/1 "Next Generation Energy-Harvesting Electronics: Holistic Approach," website: www.holistic.ecs.soton.ac.uk

\section{References}

[1] The EH Network Data Repository [Online]. Available: http://eh-network.org/data last accessed April 2012.

[2] Energy Harvesting Systems: A Block Diagram. Holistic Energy Harvesting [Online]. Available: http://www.holistic.ecs.soton.ac.uk/res/eh-system.php, last accessed April 2012.

[3] I. Ayala Garcia, D. Zhu, J. Tudor, and S. Beeby, A tunable kinetic energy harvester with dynamic over range protection, Smart Materials and Structures, 19 (11). pp. 1-10, (2010).

[4] S. Lebherz, Effective and Simple Condition Based Maintenance, Ship \& Offshore, (2010).

[5] R.K. Mobley, An Introduction to Predictive Maintenance, Butterworth-Heinemann, (2002).

[6] D. Zhu, S. Beeby, M. Tudor, and N. Harris, A credit card sized self powered smart sensor node. Sensors and Actuators A: Physical, 169(2), pp. 317-325, (2011).

[7] D. Zhu, M. Tudor, John and Beeby, Steve, Strategies for increasing the operating frequency range of vibration energy harvesters: a review, Measurement Science and Technology, 21(2), 022001, (2010). 